Published online 2017 April 13.

Abstract

\title{
The Role of Imaging in Childhood Osteomyelitis
}

\section{Roxana Azma ${ }^{1, *}$}

${ }^{1}$ Assistant Professor of Radiology, Mofid children's hospital, Shahid Beheshti University of Medical Sciences, Tehran, Iran

"Corresponding author: Roxana Azma, MD, Assistant Professor of Radiology, Mofid children's hospital, Shahid Beheshti University of Medical Sciences, Tehran, Iran. E-mail: roxanaazma@gmail.com

Received 2016 December 21; Accepted 2017 February 08.

\begin{abstract}
Osteomyelitis in childhood can be a challenging diagnosis. Imaging plays an important role in the diagnosis of osteomyelitis in children, and the imaging findings are crucial in the treatment decision. Pediatric hematogenous osteomyelitis may have variable radiological expression, which is influenced by the age and condition of the patient, the virulence of the causative agent, the stage of the disease, or the selected imaging tool. Different imaging modalities exist for imaging of osteomyelitis which include: conventional radiography, Three-phase bone scintigraphy, ultrasound (US), computed tomography (CT) and magnetic resonance (MR) imaging. Conventional radiography is the initial step in evaluation of osseous changes. In most cases it will be the only imaging technique used in the diagnosis, as well as treatment. Ultrasonography, is a very useful modality. It allows correlating the physical exam with US findings and comparing the affected side with the opposite side. After conventional imaging MRI is the most important imaging modality for the evaluation of osteomyelitis. MRI excellent delineation of the osseous component as well as, soft tissue extension, joint effusion and complications of osteomyelitis such as abscess formation, especially in case of failure to respond to therapy. CT scan is more useful and superior to MR in detecting cortical destruction, air and sequesters. Bone scintigraphy using ${ }^{99}$ Tc methylene diphosphonate may help to confirm suspected osteomyelitis by showing increased uptake in all three phases of the dynamic perfusion.
\end{abstract}

This is an abstract presented in the 33rd Iranian congress of radiology (ICR) and the 15th congress of Iranian radiographic science association (IRSA). 